\title{
ESSENTIAL BOUNDARY POINTS
}

\author{
BY WALTER RUDIN ${ }^{1}$
}

Communicated December 5, 1963

Let $B(D)$ denote the class of all bounded holomorphic functions on the connected open subset $D$ of the Riemann sphere, and call a boundary point $z_{0}$ of $D$ removable if every $f \in B(D)$ has a holomorphic extension to an open set which contains $D$ and $z_{0}$. Boundary points which are not removable are called essential. It is known [4] that if $z_{0}$ is an essential boundary point of $D$, then there exists $f \in B(D)$, with $\|f\|_{D}=1$, whose cluster set at $z_{0}$ is the entire closed unit disc. (The symbol $\|f\|_{S}$ denotes the supremum of the numbers $|f(z)|$ as $z$ ranges over the set $S$.) Thus, from one standpoint at least, it appears that every essential boundary point $z_{0}$ of $D$ has associated with it some $f \in B(D)$ whose singularity at $z_{0}$ is as bad as a singularity can be at any boundary point.

Nevertheless, there are situations in which a set of essential boundary points has many of the properties that are usually associated with interior points. The following construction illustrates this.

Let $E$ be a nonempty compact subset of the real axis $R$, subject to only one condition: we require that $m(E)=0$, where $m$ denotes one-dimensional Lebesgue measure. Let $\lambda_{0}, \lambda_{1}, \lambda_{2}, \cdots$ be positive numbers such that $\lambda_{0}<1, \lambda_{k} \rightarrow \infty$, and

$$
\sum_{k=1}^{\infty}\left(k \lambda_{k}\right)^{-1}=\infty .
$$

Let $z_{n}=x_{n}+i y_{n}(n=1,2,3, \cdots)$ be points in the open upper halfplane, located so that the set of all limit points of $\left\{z_{n}\right\}$ is precisely $E$, and put

$$
\alpha_{n}=\inf \left\{\lambda_{0}, y_{n} \lambda_{1},\left(y_{n} \lambda_{2}\right)^{2},\left(y_{n} \lambda_{3}\right)^{3}, \cdots\right\} .
$$

Since $\lambda_{k} \rightarrow \infty$, we have $\alpha_{n}>0$, and we can therefore choose $r_{n}$ so that

$$
0<r_{n}<2^{-n} y_{n} \alpha_{n}
$$

and so that the closed circular discs $\Delta_{n}$ with radius $r_{n}$ and center at $z_{n}+i r_{n}$ are disjoint; (2) and (3) imply that

$$
\sum_{n=1}^{\infty} y_{n}^{-k-1} r_{n} \leqq \begin{cases}\lambda_{0} & \text { if } k=0, \\ \lambda_{k}^{k} & \text { if } k=1,2,3, \cdots .\end{cases}
$$

\footnotetext{
${ }^{1}$ Sponsored by NSF Grant GP-2235.
} 
Finally, let $D$ be the complement of

$$
E \cup \Delta_{1} \cup \Delta_{2} \cup \Delta_{3} \cup \cdots,
$$

and let $\Gamma_{n}$ be the boundary of $\Delta_{n}$. Note that $\infty \in D$.

Theorem I. If $D$ is constructed as above, then $D$ has the following properties:

(i) Every point of $E$ is an essential boundary point of $D$.

(ii) Every $f \in B(D)$ can be extended to $D \cup E$ by the Cauchy formula

$$
f(z)=f(\infty)+\sum_{n=1}^{\infty} \frac{1}{2 \pi i} \int_{\Gamma_{n}} \frac{f(w)}{w-z} d w,
$$

and the derivatives $f^{(k)}$ can be extended to $D \cup E$ by

$$
f^{(k)}(z)=\sum_{n=1}^{\infty} \frac{k !}{2 \pi i} \int_{\Gamma_{n}} \frac{f(w)}{(w-z)^{k+1}} d w .
$$

(iii) The series (6) and (7) converge absolutely and uniformly in the closed lower half-plane $G$, and the inequalities

$$
\left\|f^{(k)}\right\|_{G} \leqq k ! \lambda_{k}^{k}|| f \|_{D} \quad(k=1,2,3, \cdots)
$$

hold. In particular, $f, f^{\prime}, f^{\prime \prime}, \ldots$ are uniformly continuous on $G$.

(iv) If $f \in B(D)$ and if $\|f\|_{E}=\|f\|_{D}$, then $f$ is constant.

(v) If $f(x)=0$ for infinitely many $x \in E$, then $f(z)=0$ for all $z \in D$.

The proofs are quite straightforward. Since every neighborhood of every point of $E$ contains some $\Delta_{n}$, we have (i). Since every $f \in B(D)$ has nontangential boundary values almost everywhere on each $\Gamma_{n}$, and since $m(E)=0$, it is easy to see that (6) and (7) hold for all $z \in D$. If $z \in G$, the absolute value of the $n$th summand in (6) is no larger than $y_{n}^{-1} r_{n}\|f\|_{D}$, the absolute value of the $n$th summand in (7) does not exceed

$$
k ! y_{n}^{-k-1} \boldsymbol{r}_{n}\|f\|_{D}
$$

and hence (ii) and (iii) follow from (4).

In particular, we have

$$
\|f\|_{E} \leqq \lambda_{0}\|f\|_{D}
$$

if $f(\infty)=0$. If $\|f\|_{D}=1$, if $f$ is not constant, and if $f(\infty)=\alpha$, we can apply (10) to the function

$$
g=\frac{f-\alpha}{1-\bar{\alpha} f}
$$

and conclude that 


$$
\|f\|_{E} \leqq \frac{\lambda_{0}+|\alpha|}{1+|\alpha| \lambda_{0}}<1
$$

This gives (iv).

The inequalities (8), combined with our assumption (1), imply that the restriction of every $f \in B(D)$ to the real axis $R$ lies in a quasianalytic class [3]. If $x_{0} \in E$ is a limit point of real zeros of $f$, then $f^{(k)}\left(x_{0}\right)=0$ for $k=0,1,2, \cdots$ (by repeated application of Rolle's theorem to the real and imaginary parts of $f$ on $R$ ). The quasianalyticity of $f$ therefore shows that $f$ vanishes on $R$, and hence on $D$, which proves (v).

Thus $E$ acts like a set of interior points as far as the Cauchy formula, the uniqueness theorem, and the maximum modulus theorem are concerned.

Let us now consider the algebra $A(D)$, consisting of all uniformly continuous holomorphic functions on $D$, which is a Banach algebra relative to the norm $\|f\|_{D}$, whose maximal ideal space is the closure $\bar{D}$ of $D$ in the Riemann sphere [1], and whose Silov boundary is

$$
\partial D=E \cup \Gamma_{1} \cup \Gamma_{2} \cup \Gamma_{3} \cup \ldots .
$$

If $E$ is countable, for instance, and if $D$ is as in Theorem $\mathrm{I},(\mathrm{v})$ shows that we obtain an example of a sup-norm algebra in which each function is determined by its values on a very small subset of the Silov boundary.

Finally, consider the so-called $\beta$-topology on the algebra $B(D)$. This was introduced by Buck [2] and is also called the "strict" topology; a typical $\beta$-neighborhood of a function $f \in B(D)$ is determined by a continuous real function $\phi$ on $\bar{D}$, positive on $D$ and 0 on $\partial D$, and it consists of all $g \in B(D)$ for which

$$
\|(g-f) \phi\|_{D}<1 \text {. }
$$

If $D$ is the unit disc, Buck has proved (unpublished) that the only $\beta$-continuous complex homomorphisms of $B(D)$ are the evaluations at points of $D$. Rubel and Shields (in a paper which is in preparation) have recently extended this to any $D$ whose boundary has no component consisting of a single point. This result cannot be extended to every $D$, however, even if every boundary point of $D$ is essential:

Theorem II. If $D$ is as in Theorem I, if $x \in E$, and if $\Phi(f)=f(x)$, then $\Phi$ is a $\beta$-continuous homomorphism of $B(D)$.

It is clear that $\Phi$ is a homomorphism, and the Cauchy formula (6), 
with $z=x$ (and with the curves $\Gamma_{n}$ replaced by nearby curves in $D$ ), shows that $\Phi(f)$ is obtained by integrating $f$ with respect to a finite measure in $D$. This shows that $\Phi$ is $\beta$-continuous [2, p. 99].

\section{REFERENCES}

1. R. Arens, The maximal ideals of certain function algebras, Pacific J. Math. 8 (1958), 641-648.

2. R. C. Buck, Bounded continuous functions on a locally compact space, Michigan Math. J. 5 (1958), 95-104.

3. T. Carleman, Sur les fonctions quasi-analytiques, Gauthier-Villars, Paris, 1926.

4. W. Rudin, Some theorems on bounded analytic functions, Trans. Amer. Math. Soc. 78 (1955), 333-342.

UNIVERSITY OF WISCONSIN 